Check for updates

Cite this: RSC Adv., 2021, 11, 30229

\title{
Microwave-assisted synthesis of 4-oxo-2-butenoic acids by aldol-condensation of glyoxylic acid $\dagger$
}

\author{
Mélanie Uguen, (D) $\ddagger^{* a}$ Conghao Gai,,$^{\text {b }}$ Lukas J. Sprenger, ${ }^{a}$ Hang Liu, \\ Andrew G. Leach (D) and Michael J. Waring (D) *a
}

4-Oxobutenoic acids are useful as biologically active species and as versatile intermediates for further derivatisation. Currently, routes to their synthesis can be problematic and lack generality. Reaction conditions for the synthesis of 4-oxo-2-butenoic acid by microwave-assisted aldol-condensation between methyl ketone derivatives and glyoxylic acid have been developed. They provide the desired products in moderate to excellent yields for a wide range of substrates, by applying a simple procedure to accessible starting materials. The investigation revealed different conditions are required depending on the nature of the methylketone substituent, with aryl derivatives proceeding best using tosic acid and aliphatic substrates reacting best with pyrrolidine and acetic acid. This substituent effect is rationalised by frontier orbital calculations. Overall, this work provides methods for synthesis of 4-oxo-butenoic acids across a broad range of substrates.

Received 19th July 2021

Accepted 1st September 2021

DOI: 10.1039/d1ra05539a

rsc.li/rsc-advances

acid-promoted, most commonly by acetic acid, ${ }^{7,9-12}$ sulphuric acid, ${ }^{13-17}$ phosphoric acid, ${ }^{13,18}$ toluene-4-sulfonic acid ${ }^{19}$ and for-

\section{Introduction}

4-Oxo-2-butenoic acids are interesting building blocks for drug discovery. Several derivatives have shown biological activity in their own right, for example to treat cancer, ${ }^{1,2}$ neurodegenerative, ${ }^{3}$ metabolic, ${ }^{4}$ and gastric ${ }^{5}$ conditions as well as antimicrobial $^{6,7}$ or antifungal ${ }^{6}$ properties (Fig. 1). In addition, their high reactivity makes them versatile intermediates for further derivatisation.

Preparation of 4-oxo-2-butenoic acids has often proven to be scope-limited, with Friedel-Crafts acylations ${ }^{1}$ used for aromatic substrates (Fig. 2) and oxidative furan-opening ${ }^{8}$ for aliphatic ones (Fig. 2). Although we managed to obtain the desired 4-oxo2-butenoic acid product when applying the oxidative furan oxidative conditions to an electron-rich aromatic example, we were unable to identify conditions compatible with electrondeficient aromatic substrates.

Aldol-condensation with glyoxylic acid is compatible with a larger range of starting materials with a few examples available in the literature, mainly using acetophenone derivatives as substrates (Fig. 2). Literature reported conditions are typically

\footnotetext{
${ }^{a}$ Cancer Research UK Drug Discovery Unit, Newcastle University Centre for Cancer, Chemistry, School of Natural and Environmental Sciences, Newcastle University, Bedson Building, Newcastle upon Tyne NE1 7RU, UK. E-mail: mike.waring@ncl.ac.uk ${ }^{b}$ Organic Chemistry Group, College of Pharmacy, Naval Medical University, Shanghai, 200433, P. R. China

${ }^{c}$ Division of Pharmacy and Optometry, School of Health Sciences, University of Manchester, Manchester M13 9PT, UK

$\dagger$ Electronic supplementary information (ESI) available. See DOI: 10.1039/d1ra05539a

\$ Joint first authors.
} mic acid, ${ }^{20}$ with two acids frequently used together. ${ }^{12,19,21-28}$ The acid promotors are usually used neat or in large excess under reflux for relatively long periods of time. A few base-promoted procedures are also described in the literature, using potassium carbonate ${ }^{29,30}$ or sodium hydroxide at reflux or under reduced pressure..$^{31}$ All these conditions are quite harsh on the reactants and resulting products, thus limiting the scope of the transformation.

Therefore, we decided to investigate the aldol-condensation with glyoxylic acid to identify efficient conditions for the preparation of 4-oxo-2-butenoic acid derivatives. We aimed to

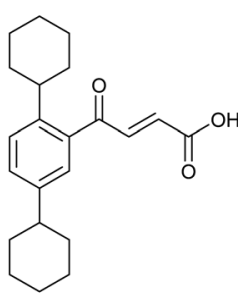

$\mathrm{IC}_{50}$ (HeLa) $=1.26 \mu \mathrm{M}$ ${ }^{\prime} C_{50}(L S 174)=4.28 \mu \mathrm{M}$ $\mathrm{IC}_{50}(\mathrm{~K} 562)=3.32 \mu \mathrm{M}$

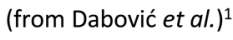

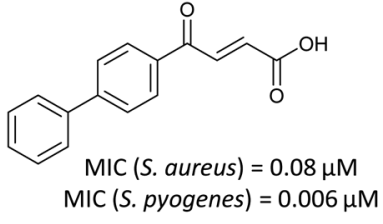

(from Nicoli et al. $)^{6}$

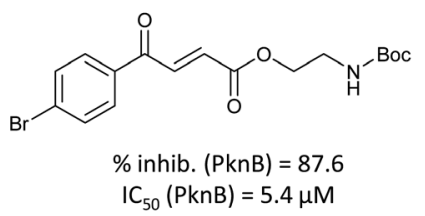

(from Wang et al.) ${ }^{7}$
Fig. 1 Examples of biologically active 4-oxo-2-butenoic acids and analogues. 


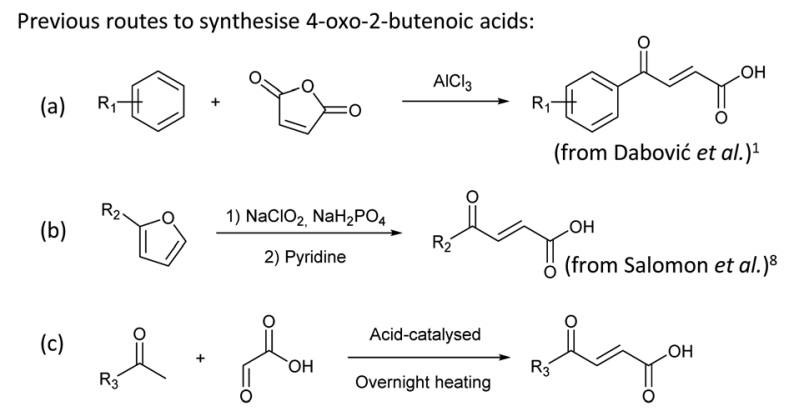

This work:

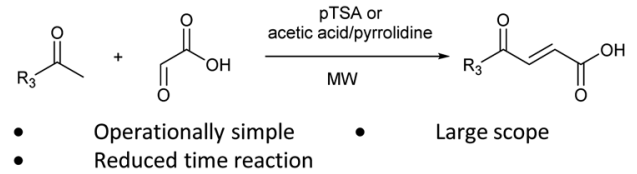

Fig. 2 Synthetic routes for the preparation of 4-oxo-2-butenoic acid derivatives.

develop operationally simple conditions compatible with a large range of substrates by using microwave heating, which had the potential to reduce reaction times and increase yields.

\section{Results and discussion}

Initially, 4-methoxyacetophenone was used as a test substrate. Reactions with glyoxylic acid under basic conditions were first attempted but showed moderate conversion to the desired product (1) (Table S1 $\dagger$ ). Reaction via the pyrrolidine-derived enamine also showed moderate conversion, with the initial aldol adduct obtained in larger amount than the desired aldol-

Table 1 Reaction conditions attempted for aldol-condensation with glyoxylic acid

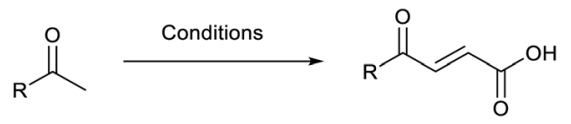

\begin{tabular}{|c|c|c|c|c|c|c|}
\hline Entry & $\mathrm{R}$ & Cond. $^{a}$ & Heat. & Time & Temp. $\left({ }^{\circ} \mathrm{C}\right)$ & Isol. yield \\
\hline 1 & & A & Conv. & $48 \mathrm{~h}$ & 80 & $70 \%$ \\
\hline 2 & & A & Conv. & $72 \mathrm{~h}$ & 80 & n.c. ${ }^{b}$ \\
\hline 3 & & A & MW & $16 \mathrm{~h}$ & 100 & $32 \%$ \\
\hline 4 & & $\mathrm{~A}$ & MW & $1 \mathrm{~h}$ & 160 & $45 \%$ \\
\hline 5 & & A & MW & $10 \mathrm{~min}$ & 180 & $<20 \%{ }^{b}$ \\
\hline 6 & & A & MW & $15 \mathrm{~min}$ & 180 & $40 \%$ \\
\hline 7 & & A & MW & $5 \mathrm{~min}$ & 160 & $0 \%$ \\
\hline 8 & & A & MW & $16 \mathrm{~h}$ & 100 & $0 \%$ \\
\hline 9 & & B & MW & $8 \mathrm{~h}$ & 80 & $25 \%$ \\
\hline 10 & & B & MW & $10 \mathrm{~min}$ & 100 & $14 \%$ \\
\hline 11 & & B & MW & $8 \mathrm{~h}$ & 60 & $52 \%$ \\
\hline
\end{tabular}

${ }^{a}$ Conditions A: 3.0 eq. glyoxylic acid monohydrate, 1.0 eq. TsOH monohydrate, dioxane; conditions B: 3.0 eq. glyoxylic acid monohydrate, 1.0 eq. pyrrolidine, 1.0 eq. acetic acid, $\mathrm{MeOH}$. ${ }^{b}$ Products not isolated. condensation product. Attempts to force the water elimination by addition of tosyl chloride led to an improved conversion of the starting material. It was proposed that the tosyl chloride was hydrolysed in situ and the reaction was catalysed by tosic acid. Accordingly, carrying out the direct tosic acid-promoted aldolcondensation provided the desired product in good yield (70\%), confirming the previous hypothesis (Table 1, entry 1). These conditions were applied to the more electron deficient 4cyanoacetophenone, in which case, even after prolonged heating, some starting ketone remained (Table 1, entry 2). To improve the conversion towards the formation of the product 2 as well as reducing the heating time, microwave-assisted heating was performed using similar conditions, leading to a moderate isolated yield of 2 (32\%, Table 1, entry 3$)$. Optimisation of the irradiation time and temperature allowed an increase of the yield to $45 \%$ with a reduced reaction time of $1 \mathrm{~h}$ (Table 1, entry 4). Shorter reaction time led to incomplete conversion of the starting material whereas increase of the temperature from $160{ }^{\circ} \mathrm{C}$ to $180{ }^{\circ} \mathrm{C}$ led to partial or total degradation of the desired product (Table 1, entries 5-7), suggesting that heating at $160{ }^{\circ} \mathrm{C}$ for 1 hour were giving the best results. These findings suggest that increased temperature and pressure allowed by the microwave reactor are key to drive the

Table 2 Scope evaluation of the aldol-condensation reaction with glyoxylic acid promoted by tosic acid ${ }^{a}$

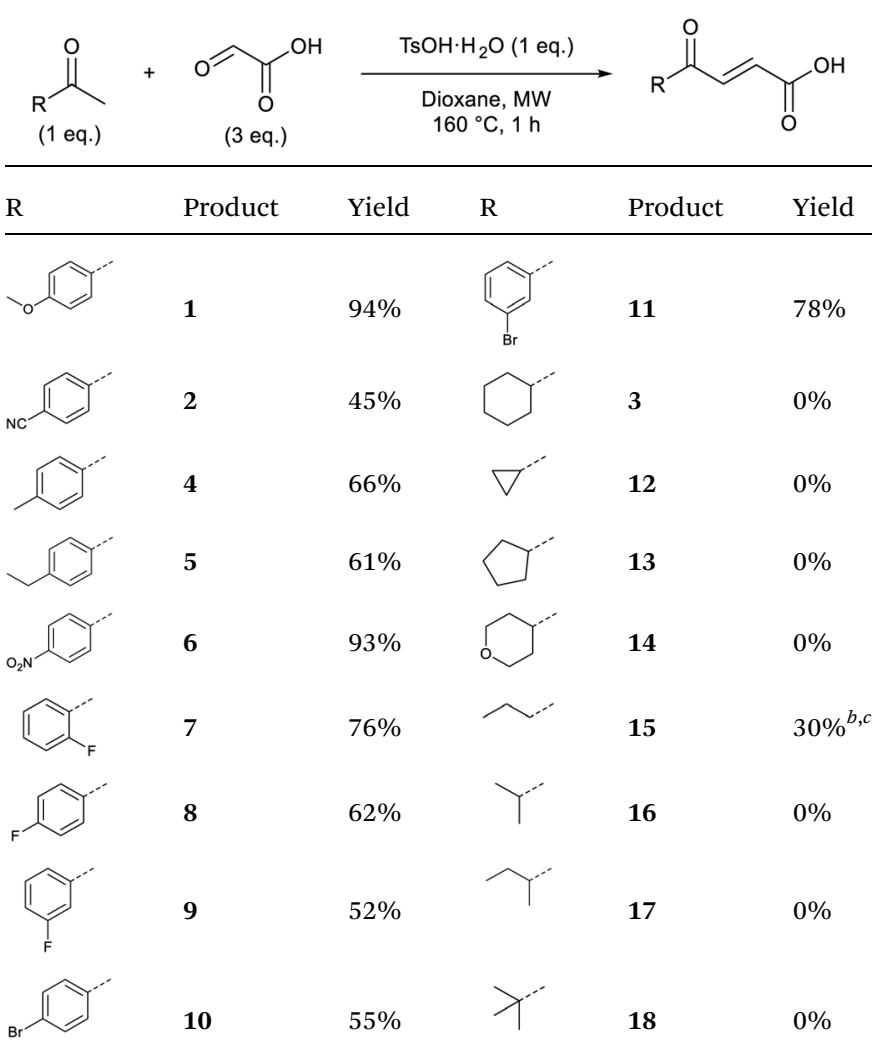

${ }^{a}$ Typical scale: $2.4 \mathrm{mmol}, 6 \mathrm{~mL}$ of solvent. ${ }^{b} \mathrm{MW}$ temperature and time: $100{ }^{\circ} \mathrm{C}, 16$ h. ${ }^{c}$ NMR yield. 
reaction while allowing a simple reaction set-up. Therefore, these optimised conditions were also applied to 4-methoxyacetophenone and showed an improved yield of 94\% (Table 2).

When applying these conditions to cyclohexylmethyl ketone starting material, no desired product 3 was formed (Table 1, entry 8). The major product was the aldol adduct intermediate. However, treatment of cyclohexylmethyl ketone with glyoxylic acid, in the presence of pyrrolidine and acetic acid (Table 1, entry 9), using microwave-assisted heating, enabled the isolation of the desired product 3 in $25 \%$ yield. Increasing the temperature from $80{ }^{\circ} \mathrm{C}$ to $100{ }^{\circ} \mathrm{C}$ led to product degradation, even with decreased reaction time (Table 1, entry 10), but decreasing the temperature to $60{ }^{\circ} \mathrm{C}$ improved the yield to $52 \%$ (Table 1 , entry 11 ).

Hence, two sets of microwave-assisted conditions for the formation of 4-oxo-2-butenoic acid by aldol-condensation of glyoxylic acid with acetyl derivative have been developed. A scope evaluation of these conditions was performed on electron-rich aromatic (1), electron-poor aromatic (2, 6-9), halogen-containing electron-neutral aromatic (10 and 11), aliphatic ring (3, 12-13), heteroatom-containing (14) and aliphatic chain (15-18) 4-oxo-2butenoic acid derivatives (Tables 2 and 3).

TsOH-promoted aldol-condensation provided the desired products in good to excellent yields for aromatic substrates generating products 1, 2 and 4-11 in $45-94 \%$ yield (Table 2).

Table 3 Scope evaluation of the aldol-condensation reaction with glyoxylic acid promoted by acetic acid and pyrrolidine ${ }^{a}$

\begin{tabular}{|c|c|c|c|c|c|}
\hline (1 eq.) & $0=\prod_{0}$ & & $\begin{array}{l}\text { cid (1 eq.) } \\
\text { ne (1 eq.) } \\
H, M W \\
C, 8 \text { h }\end{array}$ & R & \\
\hline \multirow[t]{10}{*}{$\mathrm{R}$} & Product & Yield & $\mathrm{R}$ & Product & Yield \\
\hline & 1 & $12 \%$ & & 11 & $<4 \%$ \\
\hline & 2 & $0 \%$ & & 3 & $52 \%$ \\
\hline & 4 & $5 \%$ & & 12 & $63 \%$ \\
\hline & 5 & $5 \%$ & & 13 & $52 \%$ \\
\hline & 6 & $0 \%$ & & 14 & $43 \%$ \\
\hline & 7 & $5 \%$ & & 15 & $0 \%$ \\
\hline & 8 & $5 \%$ & & 16 & $71 \%$ \\
\hline & 9 & $4 \%$ & & 17 & $92 \%$ \\
\hline & 10 & $4 \%$ & & 18 & $0 \%$ \\
\hline
\end{tabular}

${ }^{a}$ Typical scale: $2.4 \mathrm{mmol}, 6 \mathrm{~mL}$ of solvent.
Electron donating and withdrawing substituents and ortho, meta and para substitution patterns were well tolerated. No desired product formation was observed when these conditions were applied to aliphatic substrates 3, 12-14 and 16-18. Pentan-2-one, however, yielded the desired product 15 under the $\mathrm{TsOH}-$ promoted conditions (by NMR) as a minor component relative to the expected internal aldol-condensation product 19 (ratio $1: 1.5$, Fig. S1 $\dagger$ ). After heating at $100{ }^{\circ} \mathrm{C}$ for 16 hours, 19 was the only product formed ( $99 \%$ yield). Hence, this suggests, as expected, that the tosic acid mediated reaction may not be compatible with methyl ketones bearing an additional enolisable centre.

The pyrrolidine-acetic acid conditions were also applied to the selected substrates (Table 3). With aliphatic substrates, these conditions yielded the desired products 3 and 12-17 with yields between $43 \%$ and $92 \%$, in which no product was obtained with the TsOH-promoted conditions. In this case, substrates with additional enolisable centres were tolerated $(\mathbf{3}, \mathbf{1 2}-\mathbf{1 7})$ and the internal aldol adduct was not observed. No desired product was obtained for the $t$-butyl ketone 18, presumably due to increased steric hindrance. Aromatic products 1, 4, 5 and 7-11 were obtained in poor 4 to $12 \%$ yields, much lower than with the $\mathrm{TsOH}$-promoted conditions. However, no product was observed for the electronpoor examples 2 and 6 nor for the aliphatic chain 15 (Table 3).

For all the examples above, the obtained 4-oxo-2-butenoic acids all had the $E$ conformation for the alkene bond confirmed by NMR. No trace of the $Z$ alkene was observed, demonstrating the stereoselectivity of this transformation.

Finally, scale-up of the synthesis of 2 from $0.69 \mathrm{mmol}$ to $6.9 \mathrm{mmol}$ demonstrated the scalability of the TsOH-promoted reaction with no change in yield. Scale up of the synthesis of 3 using the pyrrolidine-acetic acid conditions from $4.0 \mathrm{mmol}$ to $7.2 \mathrm{mmol}$ gave increased yield (from $38 \%$ to $52 \%$ ).

Based on the assumption that the reaction proceeds by attack of the protonated glyoxylic acid by the enol form of the

Enol-mediated mechanism:

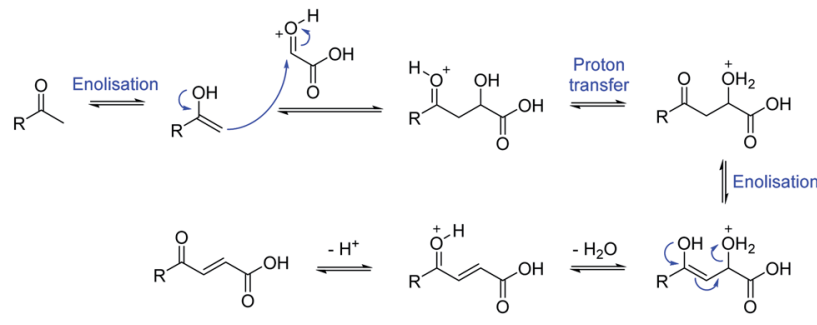

Enamine-mediated mechanism:
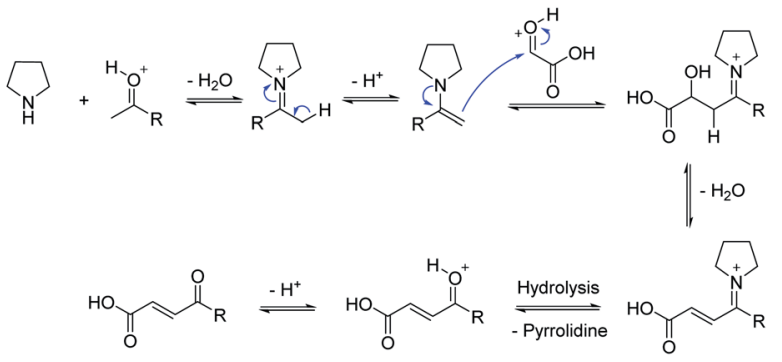

Scheme 1 Mechanisms for the aldol condensation reaction. 


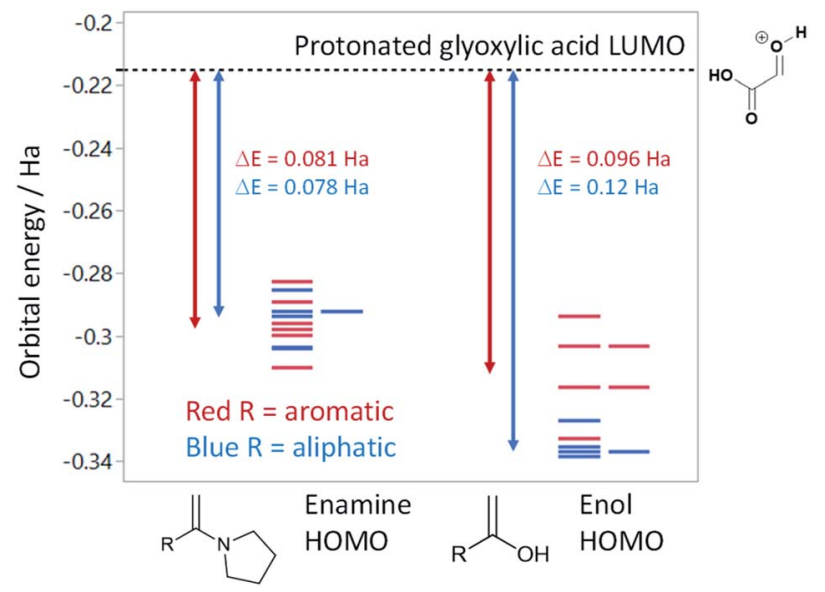

Fig. 3 Calculated HOMO energies at the RHF/6-31+G** level for selected enamines and enols relative to the protonated glyoxylic acid LUMO. Double-headed arrows show the average HOMO-LUMO gap for aromatic (red) and aliphatic (blue) substituents.

methylketone under the tosic acid promoted conditions, and by either the enol or the enamine in the presence of pyrrolidine/ acetic acid (Scheme 1) as the rate determining step, the observed differences in reactivity were rationalised from the calculated energy gaps between the protonated glyoxylic acid LUMO and the enol or enamine HOMOs (Fig. 3). Calculations employed the RHF/6-31+G** level of theory in the Gaussian09 suite of programs..$^{32}$ Geometries were optimised and frequencies computed to verify that they are minima.

HOMOs of the enol form of the aromatic ketones are consistently higher in energy than those of the aliphatic examples. This explains why the TsOH conditions work better on aromatic compounds as the HOMO-LUMO energy gap is significantly reduced $(\Delta E$ (aromatic $)=0.096 \mathrm{Ha}, \Delta E$ (aliphatic $)=0.12 \mathrm{Ha}, \Delta \Delta E$ $=0.024 \mathrm{Ha}$ ). For the aliphatic examples, HOMOs of the enamines are higher in energy compared to their corresponding enols (average HOMO-LUMO $\Delta E=0.078 \mathrm{Ha}$ ). This provides an explanation as to why the pyrrolidine-acetic acid conditions work better for aliphatic substrates, and that these reactions likely proceed via the enamine as the predominant pathway.

\section{Conclusions}

We successfully identified two sets of conditions that provide $E$-4oxo-2-butenoic acids using microwave-assisted aldol-condensation with glyoxylic acid. These provide the desired products in good to excellent yields for substrate with various electronic and steric properties (electron-rich, -neutral and -poor aromatic, ortho-, meta-, and para-substituted aromatic, linear and cyclic aliphatic). The reaction can be scaled-up to mmol scale with no effect on the yield. It was observed that tosic acid promoted conditions work better for aromatic substrates whereas acid acetic and pyrrolidine are preferred for aliphatic substrates. These findings can be rationalised by the relative differences in HOMO-LUMO energy gaps for the enol and enamine intermediates. Overall, we describe a convenient and efficient synthesis for 4-oxo-2-butenoic acids which should allow an easier access to these biologically-relevant molecules and their derivatives.

\section{Experimental}

\section{General procedure $A$ for the synthesis of 1, 2 and 4-11}

In a Biotage microwave vial, acetyl derivative (1 eq.), glyoxylic acid monohydrate ( 3 eq.) and $\mathrm{TsOH}$ monohydrate (1 eq.) were dissolved in dioxane $\left(2.5 \mathrm{~mL} \mathrm{mmol}^{-1}\right)$. The vial was closed with a $20 \mathrm{~mm}$ aluminium crimp cap with a PTFE/silicone septum and heated in the microwave for $1 \mathrm{~h}$ at $160{ }^{\circ} \mathrm{C}$ using the low absorption mode while stirred at $600 \mathrm{rpm}$ with a PTFE stirring bar. $2 \mathrm{M} \mathrm{HCl}$ aqueous solution was added to the mixture. This was extracted 3 times with $\mathrm{CH}_{2} \mathrm{Cl}_{2}$. Combined organic phases were dried over $\mathrm{MgSO}_{4}$. The solvent was removed under vacuum. A typical scale was $2.4 \mathrm{mmol}$ but the reaction was successfully scaled up and down.

(E)-4-(4-Methoxyphenyl)-4-oxobut-2-enoic acid (1). Compound 1 was obtained following General procedure A. Flash chromatography (0 to $10 \% 0.1 \mathrm{AcOH}$ in $\mathrm{MeOH}$ in $\mathrm{CH}_{2} \mathrm{Cl}_{2}$ ) provided 1 as a bright yellow solid $(2.59 \mathrm{~g}, 94 \%) . R_{\mathrm{f}}=0.65(80 \%$ EtOAc in PE); $\mathrm{mp}=180-182{ }^{\circ} \mathrm{C}$ (from MeOH); UV $\lambda_{\max }(\mathrm{EtOH} /$ nm) 287.2, 223.6, 200.0; FTIR $\left(\mathrm{cm}^{-1}\right) \nu_{\max } 2840 \mathrm{br}(\mathrm{O}-\mathrm{H}$ acid$)$, 1699s ( $\mathrm{C}=\mathrm{O}$ acid), 1661s ( $\mathrm{C}=\mathrm{O}$ ketone), 1592s $(\mathrm{C}=\mathrm{C}$ alkene), $1511 \mathrm{~s}$ ( $\mathrm{C}=\mathrm{C}$ aromatic), 1420s (O-H acid), 1167 (s, C-O methoxy); ${ }^{1} \mathrm{H}$ NMR $\left(500 \mathrm{MHz} ; \mathrm{CDCl}_{3} ; \mathrm{Me}_{4} \mathrm{Si}\right) \delta_{\mathrm{H}} 3.90(3 \mathrm{H}, \mathrm{s}$, $\left.\mathrm{CH}_{3}\right), 6.88(1 \mathrm{H}, \mathrm{d}, J=15.5 \mathrm{~Hz}, \mathrm{CH}=\mathrm{CH}), 6.97-7.02(2 \mathrm{H}, \mathrm{m}, \mathrm{CH}-$ $\mathrm{Ar}), 7.97-8.05$ (3H, $\mathrm{m}, \mathrm{CH}=\mathrm{CH}$ and $\mathrm{CH}-\mathrm{Ar}) ;{ }^{13} \mathrm{C} \mathrm{NMR}(126 \mathrm{MHz}$; $\left.\mathrm{CDCl}_{3} ; \mathrm{Me}_{4} \mathrm{Si}\right) \delta 55.77\left(\mathrm{CH}_{3}\right), 114.39(\mathrm{C}-\mathrm{Ar}), 129.68(\mathrm{C}-\mathrm{Ar}), 130.61$ $(\mathrm{C}=\mathrm{C}), 131.55$ (C-Ar), $138.77(\mathrm{C}=\mathrm{C}), 164.57$ (C-Ar), 169.84 (COOH), 187.48 (CO); MS(ES+) $\mathrm{m} / \mathrm{z}$ 207.2; HRMS calcd for $\mathrm{C}_{11} \mathrm{H}_{10} \mathrm{O}_{4}[\mathrm{M}+\mathrm{H}]^{+}$207.0579, found 207.0555.<smiles>COc1ccc(C(=O)/C=C/C(=O)O)cc1</smiles>

(E)-4-(4-Cyanophenyl)-4-oxobut-2-enoic acid (2). Compound 2 was obtained following General procedure A. Normal phase flash chromatography ( 0 to $10 \% 0.1 \% \mathrm{AcOH}$ in $\mathrm{MeOH}$ in $\mathrm{CH}_{2} \mathrm{Cl}_{2}$ ) yielded compounds 2 as a pale yellow solid (443 mg, $32 \%) . R_{\mathrm{f}}=0.12\left(10 \% \mathrm{MeOH}\right.$ in $\left.\mathrm{CH}_{2} \mathrm{Cl}_{2}\right) ; \mathrm{mp}=134-140^{\circ} \mathrm{C}$ (from $\mathrm{MeOH}) ; \mathrm{UV} \lambda_{\max }(\mathrm{EtOH} / \mathrm{nm}) 256.4$; FTIR $\left(\mathrm{cm}^{-1}\right) \nu 3063 \mathrm{br}(\mathrm{O}-\mathrm{H}$ acid), 2231w (CN); ${ }^{1} \mathrm{H}$ NMR (500 MHz; MeOD; $\left.\mathrm{Me}_{4} \mathrm{Si}\right) \delta_{\mathrm{H}} 6.83$ $(1 \mathrm{H}, \mathrm{d}, J=15.6 \mathrm{~Hz}, \mathrm{CH}=\mathrm{CH}), 7.91(1 \mathrm{H}, \mathrm{d}, J=15.6 \mathrm{~Hz}, \mathrm{CH}=\mathrm{CH})$, $7.93(2 \mathrm{H}, \mathrm{d}, J=8.4 \mathrm{~Hz}, \mathrm{CH}-\mathrm{Ar}), 8.17(2 \mathrm{H}, \mathrm{d}, J=8.4 \mathrm{~Hz}, \mathrm{CH}-\mathrm{Ar})$; ${ }^{13} \mathrm{C}$ NMR (methanol- $\left.d_{4}, 126 \mathrm{MHz}\right) \delta_{\mathrm{C}} 117.86(\mathrm{CN}), 118.87(\mathrm{C}-\mathrm{Ar})$, 130.43 (C-Ar), 133.93 (C-Ar), 135.05 (C=C), 136.84 (C=C), 141.19 (C-Ar), $168.23(\mathrm{COOH}), 190.30(\mathrm{CO}) ; \mathrm{MS}(\mathrm{ES}+) \mathrm{m} / z=201.1$ $[\mathrm{M}-\mathrm{H}]^{-}$; HRMS calcd for $\mathrm{C}_{11} \mathrm{H}_{7} \mathrm{NO}_{3} 200.0348[\mathrm{M}+\mathrm{H}]^{+}$found 200.0363.<smiles>N#Cc1ccc(C(=O)/C=C/C(=O)O)cc1</smiles> 
(E)-4-Oxo-4-(p-tolyl)but-2-enoic acid (4). Compound 4 was obtained following General procedure A. Flash chromatography ( 0 to $50 \%$ EtOAc in petroleum ether) yielded 4 as a yellow solid (300 mg, 66\%). $R_{\mathrm{f}}=0.39\left(10 \% \mathrm{MeOH}\right.$ in $\left.\mathrm{CH}_{2} \mathrm{Cl}_{2}\right)$; $\mathrm{mp}=134-$ $136{ }^{\circ} \mathrm{C}$ (from $\mathrm{MeOH}$ ); UV $\lambda_{\max }(\mathrm{EtOH} / \mathrm{nm})$ 280.0, 231.1; FTIR $\left(\mathrm{cm}^{-1}\right) \nu_{\max } 3031 \mathrm{br}(\mathrm{OH}$ acid), 2923s (CH), 1664s (C=O acid), $1410 \mathrm{~s}\left(\mathrm{O}-\mathrm{H}\right.$ acid); ${ }^{1} \mathrm{H}$ NMR (600 MHz; DMSO- $\left.d_{6} ; \mathrm{Me}_{4} \mathrm{Si}\right) \delta_{\mathrm{H}} 2.40$ $\left(3 \mathrm{H}, \mathrm{s}, \mathrm{CH}_{3}\right), 6.67(1 \mathrm{H}, \mathrm{d}, J=15.6 \mathrm{~Hz}, \mathrm{CH}=\mathrm{CH}), 7.38(2 \mathrm{H}, \mathrm{d}, J=$ $7.8 \mathrm{~Hz}, \mathrm{CH}-\mathrm{Ar}), 7.87(1 \mathrm{H}, \mathrm{d}, J=15.6 \mathrm{~Hz}, \mathrm{CH}=\mathrm{CH}), 7.94(2 \mathrm{H}, \mathrm{d}, J$ $=7.8 \mathrm{~Hz}, \mathrm{CH}-\mathrm{Ar}), 13.14$ (1H, br s, COOH); ${ }^{13} \mathrm{C}$ NMR $(150 \mathrm{MHz}$; DMSO- $\left.d_{6} ; \mathrm{Me}_{4} \mathrm{Si}\right) \delta_{\mathrm{C}} 21.67\left(\mathrm{CH}_{3}\right), 129.34$ (C-Ar), $130.04(\mathrm{C}-\mathrm{Ar})$, $133.09(\mathrm{C}=\mathrm{C}), 134.18$ (C-Ar), $136.65(\mathrm{C}=\mathrm{C}), 145.10$ (C-Ar), 166.78 (COOH), 189.30 (CO); $\mathrm{MS}\left(\mathrm{ES}^{+}\right) \mathrm{m} / \mathrm{z} 191.1[\mathrm{M}+\mathrm{H}]^{+}$; HRMS calcd for $\mathrm{C}_{11} \mathrm{H}_{11} \mathrm{O}_{3}[\mathrm{M}+\mathrm{H}]^{+}$191.0629, found 191.0697 .

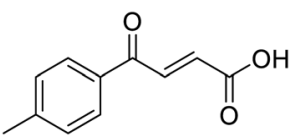

(E)-4-(4-Ethylphenyl)-4-oxobut-2-enoic acid (5). Compound 5 was obtained following General procedure A. Flash chromatography ( 0 to $50 \%$ EtOAc in petroleum ether) yielded 5 as a yellow solid (300 mg, 61\%). $R_{\mathrm{f}}=0.42\left(10 \% \mathrm{MeOH}\right.$ in $\left.\mathrm{CH}_{2} \mathrm{Cl}_{2}\right)$; $\mathrm{mp}=91-93{ }^{\circ} \mathrm{C}$ (from $\left.\mathrm{MeOH}\right) ; \mathrm{UV} \lambda_{\max }(\mathrm{EtOH} / \mathrm{nm}) 282.0,232.1$; FTIR $\left(\mathrm{cm}^{-1}\right) \nu_{\max } 3052 \mathrm{br}(\mathrm{OH}$ acid), 2968s $(\mathrm{CH}), 2934 \mathrm{~s}(\mathrm{CH})$, $1666 \mathrm{~s}$ (C=O acid), 1415s (O-H acid); ${ }^{1} \mathrm{H}$ NMR (600 MHz; DMSO$\left.d_{6} ; \mathrm{Me}_{4} \mathrm{Si}\right) \delta_{\mathrm{H}} 1.20\left(3 \mathrm{H}, \mathrm{t}, J=7.2 \mathrm{~Hz}, \mathrm{CH}_{3}\right), 2.70(2 \mathrm{H}, \mathrm{q}, J=7.2 \mathrm{~Hz}$, $\left.\mathrm{CH}_{2}\right), 6.68(1 \mathrm{H}, \mathrm{d}, J=15.6 \mathrm{~Hz}, \mathrm{CH}=\mathrm{CH}), 7.41(2 \mathrm{H}, \mathrm{d}, J=7.8 \mathrm{~Hz}$, $\mathrm{CH}-\mathrm{Ar}), 7.87$ (1H, d, $J=15.6 \mathrm{~Hz}, \mathrm{CH}=\mathrm{CH}), 7.96(2 \mathrm{H}, \mathrm{d}, J=$ $7.8 \mathrm{~Hz}, \mathrm{CH}-\mathrm{Ar}), 13.15$ (1H, br s, COOH); ${ }^{13} \mathrm{C}$ NMR $(150 \mathrm{MHz}$; DMSO- $\left.d_{6} ; \mathrm{Me}_{4} \mathrm{Si}\right) \delta_{\mathrm{C}} 15.48\left(\mathrm{CH}_{3}\right), 28.68\left(\mathrm{CH}_{2}\right), 128.87(\mathrm{C}-\mathrm{Ar})$, 129.45 (C-Ar), $133.18(\mathrm{C}=\mathrm{C}), 134.43$ (C-Ar), $136.62(\mathrm{C}=\mathrm{C})$, 151.07 (C-Ar), 166.80 (COOH), 189.34 (CO); $\mathrm{MS}\left(\mathrm{ES}^{+}\right) \mathrm{m} / \mathrm{z} 205.1$ $[\mathrm{M}+\mathrm{H}]^{+}$; HRMS calcd for $\mathrm{C}_{12} \mathrm{H}_{13} \mathrm{O}_{3}[\mathrm{M}+\mathrm{H}]^{+}$205.0791, found 205.0865 .

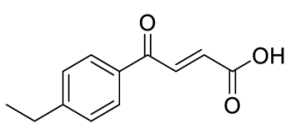

(E)-4-(4-Nitrophenyl)-4-oxobut-2-enoic acid (6). Compound 6 was obtained following General procedure A. Flash chromatography (0 to 50\% EtOAc in petroleum ether) yielded 6 as a yellow solid (493 mg, 93\%). $R_{\mathrm{f}}=0.40\left(10 \% \mathrm{MeOH}\right.$ in $\left.\mathrm{CH}_{2} \mathrm{Cl}_{2}\right)$; $\mathrm{mp}=173-175^{\circ} \mathrm{C}$ (from $\left.\mathrm{MeOH}\right) ; \mathrm{UV} \lambda_{\max }(\mathrm{EtOH} / \mathrm{nm}) 269.0$; FTIR $\left(\mathrm{cm}^{-1}\right) \nu_{\max } 2989 \mathrm{br}\left(\mathrm{OH}\right.$ acid), 1604s (C=O acid), 1530s $\left(\mathrm{NO}_{2}\right)$, 1419s (O-H acid), 1350s $\left(\mathrm{NO}_{2}\right) ;{ }^{1} \mathrm{H}$ NMR (600 MHz; DMSO- $d_{6}$; $\left.\mathrm{Me}_{4} \mathrm{Si}\right) \delta_{\mathrm{H}} 6.71(1 \mathrm{H}, \mathrm{d}, J=15.6 \mathrm{~Hz}, \mathrm{CH}=\mathrm{CH}), 7.87(1 \mathrm{H}, \mathrm{d}, J=$ $15.6 \mathrm{~Hz}, \mathrm{CH}=\mathrm{CH}), 8.25(2 \mathrm{H}, \mathrm{d}, J=9.0 \mathrm{~Hz}, \mathrm{CH}-\mathrm{Ar}), 8.36(2 \mathrm{H}, \mathrm{d}, J$ $=9.0 \mathrm{~Hz}, \mathrm{CH}-\mathrm{Ar}), 13.25$ (1H, br s, COOH); ${ }^{13} \mathrm{C}$ NMR $(150 \mathrm{MHz}$; DMSO- $\left.d_{6} ; \mathrm{Me}_{4} \mathrm{Si}\right) \delta_{\mathrm{C}} 124.42(\mathrm{C}-\mathrm{Ar}), 130.65(\mathrm{C}-\mathrm{Ar}), 134.36(\mathrm{C}=\mathrm{C})$, $136.23(\mathrm{C}=\mathrm{C}), 141.23$ (C-Ar), $150.63(\mathrm{C}-\mathrm{Ar}), 166.56(\mathrm{COOH})$, 189.40 (CO); $\mathrm{MS}\left(\mathrm{ES}^{+}\right) \mathrm{m} / \mathrm{z} 222.1[\mathrm{M}+\mathrm{H}]^{+}$; HRMS calcd for $\mathrm{C}_{10} \mathrm{H}_{6} \mathrm{NO}_{5}[\mathrm{M}-\mathrm{H}]^{-}$220.0329, found 220.0257 .<smiles>O=C(O)/C=C/C(=O)c1ccc([N+](=O)[O-])cc1</smiles>

(E)-4-(2-Fluorophenyl)-4-oxobut-2-enoic acid (7). Compound 7 was obtained following General procedure A. Flash chromatography ( 0 to $50 \%$ EtOAc in petroleum ether) yielded 7 as a yellow solid (352 mg, 76\%). $R_{\mathrm{f}}=0.41\left(10 \% \mathrm{MeOH}\right.$ in $\left.\mathrm{CH}_{2} \mathrm{Cl}_{2}\right)$; $\mathrm{mp}=105-106^{\circ} \mathrm{C}$ (from MeOH); UV $\lambda_{\max }(\mathrm{EtOH} / \mathrm{nm}) 246.0$; FTIR $\left(\mathrm{cm}^{-1}\right) \nu_{\max } 3001 \mathrm{br}(\mathrm{OH}$ acid), 1665s (C=O acid), 1421s (O-H acid); ${ }^{1} \mathrm{H}$ NMR (600 MHz; DMSO- $\left.d_{6} ; \mathrm{Me}_{4} \mathrm{Si}\right) \delta_{\mathrm{H}} 6.62(1 \mathrm{H}, \mathrm{dd}, J=$ $\left.15.6 \mathrm{~Hz}, J_{\mathrm{HF}}=0.6 \mathrm{~Hz}, \mathrm{CH}=\mathrm{CH}\right), 7.37-7.41(2 \mathrm{H}, \mathrm{m}, \mathrm{CH}-\mathrm{Ar}), 7.57$ $\left(1 \mathrm{H}, \mathrm{dd}, J=15.6 \mathrm{~Hz}, J_{\mathrm{HF}}=3.0 \mathrm{~Hz}, \mathrm{CH}=\mathrm{CH}\right), 7.70-7.74(1 \mathrm{H}, \mathrm{m}$, CH-Ar), 7.80-7.83 (1H, m, CH-Ar), 13.21 (1H, br s, COOH); ${ }^{13} \mathrm{C}$ NMR (150 MHz; DMSO- $d_{6}$; $\left.\mathrm{Me}_{4} \mathrm{Si}\right) \delta_{\mathrm{C}} 117.30\left(\mathrm{~d}, J_{\mathrm{CF}}=22.2 \mathrm{~Hz}, \mathrm{C}-\right.$ $\mathrm{Ar}), 125.50$ (d, $\left.J_{\mathrm{CF}}=2.8 \mathrm{~Hz}, \mathrm{C}-\mathrm{Ar}\right), 125.54$ (d, $J_{\mathrm{CF}}=16.6 \mathrm{~Hz}, \mathrm{C}-\mathrm{Ar}$ ), $131.22(\mathrm{C}=\mathrm{C}), 133.33(\mathrm{C}=\mathrm{C}), 136.06\left(\mathrm{~d}, J_{\mathrm{CF}}=9.0 \mathrm{~Hz}, \mathrm{C}-\mathrm{Ar}\right)$, $139.14\left(\mathrm{~d}, J_{\mathrm{CF}}=5.1 \mathrm{~Hz}, \mathrm{C}-\mathrm{Ar}\right), 161.40\left(\mathrm{~d}, J_{\mathrm{CF}}=252.0 \mathrm{~Hz}, \mathrm{CF}-\right.$ $\mathrm{Ar}), 166.63(\mathrm{COOH}), 188.54$ (CO); 19F NMR (282 MHz, DMSO$\left.d_{6}\right) \delta_{\mathrm{F}}-111.29 ; \mathrm{MS}\left(\mathrm{ES}^{+}\right) \mathrm{m} / z 195.1[\mathrm{M}+\mathrm{H}]^{+}$; HRMS calcd for $\mathrm{C}_{10} \mathrm{H}_{8} \mathrm{FO}_{3}[\mathrm{M}+\mathrm{H}]^{+}$195.0379, found 195.0452.<smiles>O=C(O)/C=C/C(=O)c1ccccc1F</smiles>

(E)-4-(4-Fluorophenyl)-4-oxobut-2-enoic acid (8). Compound 8 was obtained following General procedure A. Flash chromatography (0 to $50 \%$ EtOAc in petroleum ether) yielded 8 as a yellow solid (289 mg, 62\%). $R_{\mathrm{f}}=0.41\left(10 \% \mathrm{MeOH}\right.$ in $\left.\mathrm{CH}_{2} \mathrm{Cl}_{2}\right)$; $\mathrm{mp}=108-109^{\circ} \mathrm{C}$ (from MeOH); UV $\lambda_{\max }(\mathrm{EtOH} / \mathrm{nm}) 243.1$; FTIR $\left(\mathrm{cm}^{-1}\right) \nu_{\max } 2978 \mathrm{br}(\mathrm{OH}$ acid), 1666s (C=O acid), 1411s (O-H acid); ${ }^{1} \mathrm{H}$ NMR (600 MHz; DMSO- $\left.d_{6} ; \mathrm{Me}_{4} \mathrm{Si}\right) \delta_{\mathrm{H}} 6.68(1 \mathrm{H}, \mathrm{d}, J=$ $15.6 \mathrm{~Hz}, \mathrm{CH}=\mathrm{CH}), 7.36-7.40(2 \mathrm{H}, \mathrm{m}, \mathrm{CH}-\mathrm{Ar}), 7.87$ (1H, d, $J=$ $15.6 \mathrm{~Hz}, \mathrm{CH}=\mathrm{CH}), 8.10-8.14(2 \mathrm{H}, \mathrm{m}, \mathrm{CH}-\mathrm{Ar}), 13.07(1 \mathrm{H}, \mathrm{br}$, $\mathrm{COOH}) ;{ }^{13} \mathrm{C}$ NMR $\left(150 \mathrm{MHz}\right.$; DMSO- $\left.d_{6} ; \mathrm{Me}_{4} \mathrm{Si}\right) \delta_{\mathrm{C}} 116.51\left(\mathrm{~d}, J_{\mathrm{CF}}\right.$ $=21.9 \mathrm{~Hz}, \mathrm{C}-\mathrm{Ar}), 132.33$ (d, $\left.J_{\mathrm{CF}}=9.6 \mathrm{~Hz}, \mathrm{C}-\mathrm{Ar}\right), 133.37$ (d, $J_{\mathrm{CF}}=$ $2.0 \mathrm{~Hz}, \mathrm{C}-\mathrm{Ar}), 133.49(\mathrm{C}=\mathrm{C}), 136.40(\mathrm{C}=\mathrm{C}), 165.90\left(\mathrm{~d}, J_{\mathrm{CF}}=\right.$ $251.6 \mathrm{~Hz}, \mathrm{CF}-\mathrm{Ar}), 166.70$ (COOH), 188.54 (CO); 19F NMR (282 MHz, DMSO- $\left.d_{6}\right) \delta_{\mathrm{F}}-104.52 ; \mathrm{MS}_{\left(\mathrm{ES}^{+}\right)} \mathrm{m} / z$ 195.1 [M + H $]^{+}$; HRMS calcd for $\mathrm{C}_{10} \mathrm{H}_{8} \mathrm{FO}_{3}[\mathrm{M}+\mathrm{H}]^{+}$195.0379, found 195.0468.<smiles>O=C(O)/C=C/C(=O)c1ccc(F)cc1</smiles>

(E)-4-(3-Fluorophenyl)-4-oxobut-2-enoic acid (9). Compound 9 was obtained following General procedure A. Flash chromatography (0 to 50\% EtOAc in petroleum ether) yielded 9 as a white solid (239 mg, 52\%). $R_{\mathrm{f}}=0.41\left(10 \% \mathrm{MeOH}\right.$ in $\left.\mathrm{CH}_{2} \mathrm{Cl}_{2}\right)$; $\mathrm{mp}=109-110{ }^{\circ} \mathrm{C}$ (from MeOH); UV $\lambda_{\max }(\mathrm{EtOH} / \mathrm{nm}) 246.0$; FTIR $\left(\mathrm{cm}^{-1}\right) \nu_{\max } 3079 \mathrm{br}(\mathrm{OH}$ acid), 1668s (C=O acid), 1416s (O-H acid); ${ }^{1} \mathrm{H}$ NMR (600 MHz; DMSO- $\left.d_{6} ; \mathrm{Me}_{4} \mathrm{Si}\right) \delta_{\mathrm{H}} 6.70(1 \mathrm{H}, \mathrm{d}, J=$ $15.6 \mathrm{~Hz}, \mathrm{CH}=\mathrm{CH}), 7.54-7.58(1 \mathrm{H}, \mathrm{m}, \mathrm{CH}-\mathrm{Ar}), 7.62-7.65(1 \mathrm{H}, \mathrm{m}$, CH-Ar), 7.79-7.81 (1H, m, CH-Ar), $7.85(1 \mathrm{H}, \mathrm{d}, J=15.6 \mathrm{~Hz}, \mathrm{CH}=$ $\mathrm{CH}), 7.90\left(1 \mathrm{H}, \mathrm{dd}, J=7.8 \mathrm{~Hz}, J_{\mathrm{HF}}=0.6 \mathrm{~Hz}, \mathrm{CH}-\mathrm{Ar}\right), 13.18(1 \mathrm{H}$, br s, COOH); ${ }^{13} \mathrm{C}$ NMR (150 MHz; DMSO- $\left.d_{6} ; \mathrm{Me}_{4} \mathrm{Si}\right) \delta_{\mathrm{C}} 115.56(\mathrm{~d}$, 
$\left.J_{\mathrm{CF}}=22.4 \mathrm{~Hz}, \mathrm{C}-\mathrm{Ar}\right), 121.35\left(\mathrm{~d}, J_{\mathrm{CF}}=21.3 \mathrm{~Hz}, \mathrm{C}-\mathrm{Ar}\right), 125.55\left(\mathrm{~d}, J_{\mathrm{CF}}\right.$ $=1.4 \mathrm{~Hz}, \mathrm{C}-\mathrm{Ar}), 131.68\left(\mathrm{~d}, J_{\mathrm{CF}}=7.8 \mathrm{~Hz}, \mathrm{C}-\mathrm{Ar}\right), 133.94(\mathrm{C}=\mathrm{C})$, $136.23(\mathrm{C}=\mathrm{C}), 138.80\left(\mathrm{~d}, J_{\mathrm{CF}}=6.2 \mathrm{~Hz}, \mathrm{C}-\mathrm{Ar}\right), 162.72\left(\mathrm{~d}, J_{\mathrm{CF}}=\right.$ $244.5 \mathrm{~Hz}, \mathrm{CF}-\mathrm{Ar}), 166.64$ (COOH), 189.02 (CO); ${ }^{19} \mathrm{~F}-\mathrm{NMR}(282$ MHz, DMSO- $\left.d_{6}\right) \delta_{\mathrm{F}}-111.78$; $\mathrm{MS}\left(\mathrm{ES}^{+}\right) \mathrm{m} / z$ 195.1 [M + H $]^{+}$; HRMS calcd for $\mathrm{C}_{10} \mathrm{H}_{6} \mathrm{FO}_{3}[\mathrm{M}-\mathrm{H}]^{-}$193.0379, found 193.0306.

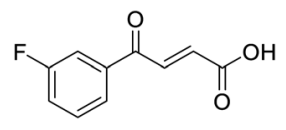

(E)-4-(4-Bromophenyl)-4-oxobut-2-enoic

acid

(10).

Compound 10 was obtained following General procedure A. Flash chromatography ( 0 to $50 \%$ EtOAc in petroleum ether) yielded 10 as a white solid (334 mg, 55\%). $R_{\mathrm{f}}=0.41(10 \% \mathrm{MeOH}$ in $\mathrm{CH}_{2} \mathrm{Cl}_{2}$ ); $\mathrm{mp}=149-152{ }^{\circ} \mathrm{C}$ (from $\left.\mathrm{MeOH}\right) ; \mathrm{UV} \lambda_{\max }(\mathrm{EtOH} / \mathrm{nm})$ 265.0; FTIR ( $\left.\mathrm{cm}^{-1}\right) \nu_{\max } 3020 \mathrm{br}(\mathrm{OH}$ acid), 1666s (C=O acid), $1418 \mathrm{~s}\left(\mathrm{O}-\mathrm{H}\right.$ acid); ${ }^{1} \mathrm{H}$ NMR (600 MHz; DMSO- $\left.d_{6} ; \mathrm{Me}_{4} \mathrm{Si}\right) \delta_{\mathrm{H}} 6.69$ (1H, d, $J=15.6 \mathrm{~Hz}, \mathrm{CH}=\mathrm{CH}), 7.78-7.81$ (2H, m, CH-Ar), 7.85 $(1 \mathrm{H}, \mathrm{d}, J=15.6 \mathrm{~Hz}, \mathrm{CH}=\mathrm{CH}), 7.96-7.98$ (2H, m, CH-Ar), 13.09 $(1 \mathrm{H}$, br s, COOH $) ;{ }^{13} \mathrm{C}$ NMR $\left(150 \mathrm{MHz} ; \mathrm{DMSO}-d_{6} ; \mathrm{Me}_{4} \mathrm{Si}\right) \delta_{\mathrm{C}}$ 128.65 (CBr-Ar), 131.21 (C-Ar), 132.55 (C-Ar), $133.78(\mathrm{C}=\mathrm{C})$, 135.64 (C-Ar), $136.26(\mathrm{C}=\mathrm{C}), 166.69$ (COOH), 189.28 (CO); $\mathrm{MS}\left(\mathrm{ES}^{+}\right) \mathrm{m} / \mathrm{z} 254.1\left[\mathrm{M}\left({ }^{79} \mathrm{Br}\right)+\mathrm{H}\right]^{+}$and $256.1\left[\mathrm{M}\left({ }^{81} \mathrm{Br}\right)+\mathrm{H}\right]^{+}$; HRMS calcd for $\mathrm{C}_{10} \mathrm{H}_{8}{ }^{79} \mathrm{BrO}_{3}[\mathrm{M}+\mathrm{H}]^{+}$254.9585, found 254.9652 .

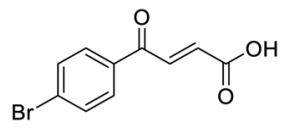

(E)-4-(3-Bromophenyl)-4-oxobut-2-enoic

acid

(11). Compound $\mathbf{1 1}$ was obtained following General procedure A. Flash chromatography ( 0 to $50 \%$ EtOAc in petroleum ether) yielded 11 as a white solid (476 mg, 78\%). $R_{\mathrm{f}}=0.41(10 \% \mathrm{MeOH}$ in $\mathrm{CH}_{2} \mathrm{Cl}_{2}$ ); $\mathrm{mp}=148-151^{\circ} \mathrm{C}$ (from $\left.\mathrm{MeOH}\right) ; \mathrm{UV} \lambda_{\max }(\mathrm{EtOH} / \mathrm{nm})$ 244.8; FTIR ( $\mathrm{cm}^{-1}$ ) $\nu_{\max } 2992 \mathrm{br}(\mathrm{OH}$ acid), 1672s ( $\mathrm{C}=\mathrm{O}$ acid), $1416 \mathrm{~s}\left(\mathrm{O}-\mathrm{H}\right.$ acid); ${ }^{1} \mathrm{H}$ NMR (600 MHz; DMSO- $\left.d_{6} ; \mathrm{Me}_{4} \mathrm{Si}\right) \delta_{\mathrm{H}} 6.70$ $(1 \mathrm{H}, \mathrm{d}, J=15.6 \mathrm{~Hz}, \mathrm{CH}=\mathrm{CH}), 7.55(1 \mathrm{H}, \mathrm{t}, J=7.8 \mathrm{~Hz}, \mathrm{CH}-\mathrm{Ar})$, $7.85(1 \mathrm{H}, \mathrm{d}, J=15.6 \mathrm{~Hz}, \mathrm{CH}=\mathrm{CH}), 7.91-7.92(1 \mathrm{H}, \mathrm{m}, \mathrm{CH}-\mathrm{Ar})$, 8.04-8.05 (1H, m, CH-Ar), 8.15-8.16 (1H, m, CH-Ar), 13.08 $(1 \mathrm{H}$, br s, COOH $) ;{ }^{13} \mathrm{C}$ NMR $\left(150 \mathrm{MHz} ; \mathrm{DMSO}-d_{6} ; \mathrm{Me}_{4} \mathrm{Si}\right) \delta_{\mathrm{C}}$ 122.81 (CBr-Ar), 128.32 (C-Ar), 131.57 (C-Ar), 131.68 (C-Ar), $134.03(\mathrm{C}=\mathrm{C}), 136.24(\mathrm{C}=\mathrm{C}), 136.95$ (C-Ar), 138.66 (C-Ar), $166.66(\mathrm{COOH}), 189.05(\mathrm{CO}) ; \mathrm{MS}\left(\mathrm{ES}^{+}\right) \mathrm{m} / z 254.1\left[\mathrm{M}\left({ }^{79} \mathrm{Br}\right)+\mathrm{H}\right]^{+}$ and $256.1\left[\mathrm{M}\left({ }^{81} \mathrm{Br}\right)+\mathrm{H}\right]^{+}$; HRMS calcd for $\mathrm{C}_{10} \mathrm{H}_{6}{ }^{79} \mathrm{BrO}_{3}[\mathrm{M}-\mathrm{H}]^{-}$ 252.9585, found 252.9503 .<smiles>O=C(O)/C=C/C(=O)c1cccc(Br)c1</smiles>

\section{General procedure $B$ for the synthesis of 3, 12-14 and 16-17}

In a Biotage microwave vial, methyl ketone-containing compound (1 eq.), glyoxylic acid monohydrate (3 eq.) and acetic acid (1 eq.) were dissolved in $\mathrm{MeOH}\left(2.5 \mathrm{~mL} \mathrm{mmol}^{-1}\right)$ then pyrrolidine (1 eq.) was added. The vial was sealed with a $20 \mathrm{~mm}$ aluminium crimp cap with PTFE/silicon septa and the mixture stirred for 5 min using a PTFE stirring bar. The mixture was irradiated using the $\mathrm{MW}$ at $60{ }^{\circ} \mathrm{C}$ for $8 \mathrm{~h}$ while stirred at $600 \mathrm{rpm}$. The solvent was removed under vacuum. A typical scale was $2.4 \mathrm{mmol}$ but the reaction was successfully scaled up and down.

(E)-4-Cyclohexyl-4-oxobut-2-enoic acid (3). Compound 3 was obtained following General procedure B. Flash chromatography ( 0 to $15 \% 0.1 \% \mathrm{AcOH}$ in $\mathrm{MeOH}$ in $\mathrm{CH}_{2} \mathrm{Cl}_{2}$ ) yielded 3 as a beige solid $(277 \mathrm{mg}, 38 \%) . R_{\mathrm{f}}=0.32\left(5 \% \mathrm{MeOH}\right.$ in $\left.\mathrm{CH}_{2} \mathrm{Cl}_{2}\right) ; \mathrm{mp}=$ 113-115 ${ }^{\circ} \mathrm{C}$ (from MeOH); UV $\lambda_{\max }(\mathrm{EtOH} / \mathrm{nm}) 330.8$, 219.8; FTIR $\left(\mathrm{cm}^{-1}\right) \nu_{\max } 3062 \mathrm{br}(\mathrm{OH}$ acid), 2922s (CH), 2851s (CH), 1660s (C=O acid), 1427s (O-H acid); 1H NMR (500 MHz; MeOD; $\left.\mathrm{Me}_{4} \mathrm{Si}\right) \delta_{\mathrm{H}} 1.05-1.21(5 \mathrm{H}, \mathrm{m}, \mathrm{Cy}), 1.51-1.67$ (5H, m, Cy), $2.50(1 \mathrm{H}$, $\mathrm{tt}, J=10.7,3.4 \mathrm{~Hz}, \mathrm{Cy}), 6.42(1 \mathrm{H}, \mathrm{d}, J=15.9 \mathrm{~Hz}, \mathrm{C}=\mathrm{C}), 6.91(1 \mathrm{H}$, $\mathrm{d}, J=15.9 \mathrm{~Hz}, \mathrm{C}=\mathrm{C}) ;{ }^{13} \mathrm{C}$ NMR $\left(126 \mathrm{MHz} ; \mathrm{MeOD} ; \mathrm{Me}_{4} \mathrm{Si}\right) \delta_{\mathrm{C}}$ 26.49 (Сyc), 29.31 (Cyc), 50.35 (Cyc), 132.21 (C=C), 139.56 (C= C), 168.50 (COOH), 204.38 (CO); $\mathrm{MS}(\mathrm{ES}+) \mathrm{m} / \mathrm{z} 183.1[\mathrm{M}+\mathrm{H}]^{+}$; HRMS calcd for $\mathrm{C}_{10} \mathrm{H}_{14} \mathrm{O}_{3}[\mathrm{M}-\mathrm{H}]^{-}$181.0870, found 181.0870.<smiles>O=C(O)/C=C/C(=O)C1CCCCC1</smiles>

(E)-4-Cyclopropyl-4-oxobut-2-enoic acid (12). Compound 12 was obtained following General procedure B. Flash chromatography (0 to 50\% EtOAc in petroleum ether) yielded 12 as a yellow oil (213 mg, 63\%). $R_{\mathrm{f}}=0.40\left(10 \% \mathrm{MeOH}\right.$ in $\left.\mathrm{CH}_{2} \mathrm{Cl}_{2}\right)$; UV $\lambda_{\max }(\mathrm{EtOH} / \mathrm{nm}) 223.0 ;$ FTIR $\left(\mathrm{cm}^{-1}\right) \nu_{\max } 3012 \mathrm{br}(\mathrm{OH}$ acid), $1715 \mathrm{~s}\left(\mathrm{C}=\mathrm{O}\right.$ acid), 1392s (O-H acid); ${ }^{1} \mathrm{H}$ NMR (600 MHz; DMSO$\left.d_{6} ; \mathrm{Me}_{4} \mathrm{Si}\right) \delta_{\mathrm{H}}$ 0.96-0.98 (2H, m, CHH-cyclopropane), 0.99-1.03 $(2 \mathrm{H}, \mathrm{m}, \mathrm{CH} H$-cyclopropane $), 2.47-2.51$ (1H, m, CHcyclopropane), $6.70(1 \mathrm{H}, \mathrm{d}, J=16.0 \mathrm{~Hz}, \mathrm{CH}=\mathrm{CH}), 7.03(1 \mathrm{H}, \mathrm{d}$, $J=16.0 \mathrm{~Hz}, \mathrm{CH}=\mathrm{CH}), 13.06(1 \mathrm{H}$, br s, COOH $) ;{ }^{13} \mathrm{C}$ NMR (150 MHz; DMSO- $\left.d_{6} ; \mathrm{Me}_{4} \mathrm{Si}\right) \delta_{\mathrm{C}} 12.07\left(\mathrm{CH}_{2}\right), 19.82(\mathrm{CH}), 131.92(\mathrm{C}=$ C), $\left.139.56(\mathrm{C}=\mathrm{C}), 167.00(\mathrm{COOH}), 200.20(\mathrm{CO}) ; \mathrm{MS} \mathrm{ES}^{+}\right) \mathrm{m} / \mathrm{z}$ $141.1[\mathrm{M}+\mathrm{H}]^{+}$; HRMS calcd for $\mathrm{C}_{7} \mathrm{H}_{8} \mathrm{O}_{3}[\mathrm{M}-\mathrm{H}]^{-}$139.0473, found 139.0400 .<smiles>O=C(O)/C=C/C(=O)C1CC1</smiles>

(E)-4-Cyclopentyl-4-oxobut-2-enoic acid (13). Compound 13 was obtained following General procedure B. Flash chromatography ( 0 to $50 \%$ EtOAc in petroleum ether) yielded 13 as a yellow oil (210 mg, 52\%). $R_{\mathrm{f}}=0.38\left(10 \% \mathrm{MeOH}\right.$ in $\left.\mathrm{CH}_{2} \mathrm{Cl}_{2}\right)$; UV $\lambda_{\max }(\mathrm{EtOH} / \mathrm{nm}) 221.0$; FTIR $\left(\mathrm{cm}^{-1}\right) \nu_{\max } 3065 \mathrm{br}(\mathrm{OH}$ acid), 2951s (CH), 2869s (CH), 1687s ( $\mathrm{C}=\mathrm{O}$ acid), 1428s (O-H acid); ${ }^{1} \mathrm{H}$ NMR (600 MHz; DMSO- $d_{6}$; $\left.\mathrm{Me}_{4} \mathrm{Si}\right) \delta_{\mathrm{H}} 1.54-1.58\left(4 \mathrm{H}, \mathrm{m}, \mathrm{CH}_{2} \mathrm{CH}_{2}-\right.$ cyclopentane), 1.64-1.85 (4H, m, $\mathrm{CH}_{2} \mathrm{CH}_{2}$-cyclopentane), 3.31 (1H, m, CH-cyclopenpane), $6.62(1 \mathrm{H}, \mathrm{d}, J=15.6 \mathrm{~Hz}, \mathrm{CH}=\mathrm{CH})$, $6.99(1 \mathrm{H}, \mathrm{d}, J=15.6 \mathrm{~Hz}, \mathrm{CH}=\mathrm{CH}), 13.09(1 \mathrm{H}$, br s, $\mathrm{COOH}) ;{ }^{13} \mathrm{C}$ NMR $\left(150 \mathrm{MHz} ; \mathrm{DMSO}-d_{6} ; \mathrm{Me}_{4} \mathrm{Si}\right) \delta_{\mathrm{C}} 26.09\left(\mathrm{CH}_{2} \mathrm{CH}_{3}\right.$-cyclopentane), $28.63\left(\mathrm{CH}_{2} \mathrm{CH}_{3}\right.$-cyclopentane), $49.34(\mathrm{CH}), 132.00$ $\left.(\mathrm{C}=\mathrm{C}), 139.26(\mathrm{C}=\mathrm{C}), 166.97(\mathrm{COOH}), 202.25(\mathrm{CO}) ; \mathrm{MS} \mathrm{ES}^{+}\right) \mathrm{m} / \mathrm{z}$ 
169.1 $[\mathrm{M}+\mathrm{H}]^{+}$; HRMS calcd for $\mathrm{C}_{9} \mathrm{H}_{12} \mathrm{O}_{3}[\mathrm{M}-\mathrm{H}]^{-}$167.0798, found 167.0725.<smiles>O=C(O)/C=C/C(=O)C1CCCC1</smiles>

(E)-4-Oxo-4-(tetrahydro-2H-pyran-4-yl)but-2-enoic acid (14). Compound 14 was obtained following General procedure B. Flash chromatography ( 0 to $10 \% 0.1 \% \mathrm{AcOH}$ in $\mathrm{MeOH}$ in $\mathrm{CH}_{2} \mathrm{Cl}_{2}$ ) yielded 14 as an orange solid (321 mg, 38\%). $R_{\mathrm{f}}=0.41(5 \% \mathrm{MeOH}$ in $\mathrm{CH}_{2} \mathrm{Cl}_{2}$ ); mp $=90-92{ }^{\circ} \mathrm{C}$ (from $\mathrm{MeOH}$ ); UV $\lambda_{\max }(\mathrm{EtOH} / \mathrm{nm}) 243.0$, 201.7; FTIR $\left(\mathrm{cm}^{-1}\right) \nu_{\max } 3067 \mathrm{br}(\mathrm{O}-\mathrm{H}$ acid), $2963(\mathrm{CH}), 2841(\mathrm{CH})$, 1669s ( $\mathrm{C}=\mathrm{O}$ acid), 1424s (O-H acid), 1117s (C-O ether); ${ }^{1} \mathrm{H}$ NMR $\left(500 \mathrm{MHz} ; \mathrm{MeOD} ; \mathrm{Me}_{4} \mathrm{Si}\right) \delta_{\mathrm{H}} 1.53(2 \mathrm{H}, \mathrm{dtd}, J=13.4,11.4,4.2 \mathrm{~Hz}$, Pyr), 1.71 (2H, ddd, $J=13.4,4.2,2.1 \mathrm{~Hz}, \mathrm{Pyr}), 2.93$ (1H, tt, $J=11.4$, $3.8 \mathrm{~Hz}, \mathrm{Pyr}), 3.42$ (2H, td, $J=11.4,2.1 \mathrm{~Hz}, \mathrm{Pyr}), 3.87$ (2H, ddd, $J=$ 11.4, 4.2, $2.1 \mathrm{~Hz}, \mathrm{Pyr}), 6.62(1 \mathrm{H}, \mathrm{d}, J=15.9 \mathrm{~Hz}, \mathrm{C}=\mathrm{C}), 7.07(1 \mathrm{H}, \mathrm{d}, J$ $=15.9 \mathrm{~Hz}, \mathrm{C}=\mathrm{C}) ;{ }^{13} \mathrm{C}$ NMR $\left(126 \mathrm{MHz} ; \mathrm{MeOD} ; \mathrm{Me}_{4} \mathrm{Si}\right) \delta_{\mathrm{C}} 29.06$ (Pyr), 47.01 (Pyr), 68.07 (Pyr), 132.75 (C=C), 139.07 (C=C), 168.44 (COOH), 202.59 (CO); MS(ES+) $m / z 185.1[\mathrm{M}+\mathrm{H}]^{+}$; HRMS calcd for $\mathrm{C}_{9} \mathrm{H}_{12} \mathrm{O}_{4}[\mathrm{M}+\mathrm{H}]^{+}$183.0663, found 183.0644.<smiles>O=C(O)/C=C/C(=O)C1CCOCC1</smiles>

(E)-5-Methyl-4-oxohex-2-enoic acid (16). Compound 16 was obtained following General procedure B. Normal phase flash chromatography (0 to $10 \% 0.1 \% \mathrm{AcOH}$ in $\mathrm{MeOH}$ in $\mathrm{CH}_{2} \mathrm{Cl}_{2}$ ) yielded compound 16 as a pale yellow oil ( $53 \mathrm{mg}, 37 \%) . R_{\mathrm{f}}=0.57(5 \%$ $\mathrm{MeOH}$ in $\left.\mathrm{CH}_{2} \mathrm{Cl}_{2}\right)$; UV $\lambda_{\text {max }}(\mathrm{EtOH} / \mathrm{nm}) 221.8$; FTIR $\left(\mathrm{cm}^{-1}\right) \nu_{\max }$ $3376 \mathrm{br}$ (O-H acid), 1686s ( $\mathrm{C}=\mathrm{O}$ acid), 1638s (C=O ketone), 1466 (O-H acid); ${ }^{1} \mathrm{H}$ NMR (500 MHz; MeOD; $\left.\mathrm{Me}_{4} \mathrm{Si}\right) \delta_{\mathrm{H}} 0.99$ (6H, d, $J=$ $6.8 \mathrm{~Hz}, \mathrm{Me}), 2.83(1 \mathrm{H}$, hept, $J=6.8 \mathrm{~Hz}, \mathrm{CH}), 6.54(1 \mathrm{H}, \mathrm{d}, J=$ $15.9 \mathrm{~Hz}, \mathrm{C}=\mathrm{C}), 7.01(1 \mathrm{H}, \mathrm{d}, J=15.9 \mathrm{~Hz}, \mathrm{C}=\mathrm{C}) ;{ }^{13} \mathrm{C}$ NMR (126 $\left.\mathrm{MHz} ; \mathrm{MeOD} ; \mathrm{Me}_{4} \mathrm{Si}\right) \delta 18.24(\mathrm{Me}), 40.57\left(\mathrm{CMe}_{2}\right), 132.44(\mathrm{C}=\mathrm{C})$, 139.36 (C=C), 168.56 (COOH), 205.15 (CO); MS(ES+) m/z 143.1 [M $+\mathrm{H}]^{+}$; HRMS calcd for $\mathrm{C}_{7} \mathrm{H}_{10} \mathrm{O}_{3}[\mathrm{M}-\mathrm{H}]^{-}$141.0557, found 141.0536 .<smiles>CC(C)C(=O)/C=C/C(=O)O</smiles>

(E)-5-Methyl-4-oxohept-2-enoic acid (17). Compound 17 was obtained following General procedure B. Flash chromatography ( 0 to $50 \%$ EtOAc in petroleum ether) yielded 17 as a yellow oil (343 mg, 92\%). $R_{\mathrm{f}}=0.39\left(10 \% \mathrm{MeOH}\right.$ in $\left.\mathrm{CH}_{2} \mathrm{Cl}_{2}\right)$; UV $\lambda_{\max }$ $(\mathrm{EtOH} / \mathrm{nm}) 272.4$; FTIR $\left(\mathrm{cm}^{-1}\right) \nu_{\max } 2968 \mathrm{br}(\mathrm{OH}$ acid), 1631s $\left(\mathrm{C}=\mathrm{O}\right.$ acid), $1454 \mathrm{~s}$ (O-H acid); ${ }^{1} \mathrm{H}$ NMR (600 MHz; DMSO- $d_{6}$; $\left.\mathrm{Me}_{4} \mathrm{Si}\right) \delta_{\mathrm{H}} 0.82\left(3 \mathrm{H}, \mathrm{t}, J=7.2 \mathrm{~Hz}, \mathrm{CH}_{2} \mathrm{CH}_{3}\right), 1.02(3 \mathrm{H}, \mathrm{d}, J=$ $\left.6.6 \mathrm{~Hz}, \mathrm{CH}_{3}\right), 1.37(1 \mathrm{H}, \mathrm{dq}, J=6.6 \mathrm{~Hz}$ and $7.2 \mathrm{~Hz}, \mathrm{CHH}), 1.37$ $(1 \mathrm{H}, \mathrm{dq}, J=6.6 \mathrm{~Hz}$ and $7.2 \mathrm{~Hz}, \mathrm{CH} H), 2.89(1 \mathrm{H}$, sext, $J=6.6 \mathrm{~Hz}$, $\mathrm{CH}), 6.62(1 \mathrm{H}, \mathrm{d}, J=15.6 \mathrm{~Hz}, \mathrm{CH}=\mathrm{CH}), 7.05(1 \mathrm{H}, \mathrm{d}, J=15.6 \mathrm{~Hz}$, $\mathrm{CH}=\mathrm{CH}), 13.10(1 \mathrm{H}$, br s, COOH $) ;{ }^{13} \mathrm{C}$ NMR (150 MHz; DMSO$\left.d_{6} ; \mathrm{Me}_{4} \mathrm{Si}\right) \delta_{\mathrm{C}} 11.61\left(\mathrm{CH}_{2} \mathrm{CH}_{3}\right), 15.59\left(\mathrm{CH}_{3}\right), 25.53\left(\mathrm{CH}_{2} \mathrm{CH}_{3}\right)$, $45.52(\mathrm{CH}), 131.93(\mathrm{C}=\mathrm{C}), 138.73(\mathrm{C}=\mathrm{C}), 166.92(\mathrm{COOH})$,
203.66 (CO); $\mathrm{MS}\left(\mathrm{ES}^{+}\right) \mathrm{m} / \mathrm{z} 157.1[\mathrm{M}+\mathrm{H}]^{+}$; HRMS calcd for $\mathrm{C}_{8} \mathrm{H}_{12} \mathrm{O}_{3}[\mathrm{M}-\mathrm{H}]^{-}$155.0786, found 155.0713.<smiles>CCC(C)C(=O)/C=C/C(=O)O</smiles>

(E)-3-Ethyl-4-oxopent-2-enoic acid (19). Compound 19 was obtained following General procedure A. Normal phase flash chromatography (0 to $10 \% 0.1 \% \mathrm{AcOH}$ in $\mathrm{MeOH}$ in $\mathrm{CH}_{2} \mathrm{Cl}_{2}$ ) yielded compound 19 as a yellow oil (132 $\mathrm{mg}, 0.93 \mathrm{mmol}, 99 \%)$. $R_{\mathrm{f}}=0.63\left(5 \% \mathrm{MeOH}\right.$ in $\left.\mathrm{CH}_{2} \mathrm{Cl}_{2}\right)$; UV $\lambda_{\max }(\mathrm{EtOH} / \mathrm{nm}) 220.9$; FTIR $\left(\mathrm{cm}^{-1}\right) \nu 3144 \mathrm{br}(\mathrm{O}-\mathrm{H}$ acid), $2974(\mathrm{CH}), 1681 \mathrm{~s}(\mathrm{C}=\mathrm{O}$ acid), 1362 (O-H acid); ${ }^{1} \mathrm{H}$ NMR (500 MHz; MeOD; $\left.\mathrm{Me}_{4} \mathrm{Si}\right) \delta_{\mathrm{H}} 0.99(3 \mathrm{H}, \mathrm{t}, J=$ $7.5 \mathrm{~Hz}, \mathrm{Me}$ ), 2.37 (3H, s, COMe), $2.71\left(2 \mathrm{H}, \mathrm{q}, J=7.5 \mathrm{~Hz}, \mathrm{CH}_{2}\right.$ ), $6.64(1 \mathrm{H}, \mathrm{s}, \mathrm{C}=\mathrm{C}) ;{ }^{13} \mathrm{C} \mathrm{NMR}\left(126 \mathrm{MHz} ; \mathrm{MeOD} ; \mathrm{Me}_{4} \mathrm{Si}\right) \delta_{\mathrm{C}} 14.06$ (Me), $20.87\left(\mathrm{CH}_{2}\right), 26.56$ (COMe), $128.17(\mathrm{C}=\mathrm{C}), 156.25(\mathrm{C}=\mathrm{C})$, 169.07 (COOH), 201.87 (CO); MS(ES+) m/z 143.1 [M + H $]^{+}$; HRMS calcd for $\mathrm{C}_{7} \mathrm{H}_{10} \mathrm{O}_{3}[\mathrm{M}-\mathrm{H}]^{-}$141.0557, found 141.0560.<smiles>CC/C(=C\C(=O)O)C(C)=O</smiles>

\section{Author contributions}

The investigation was performed by M. U., C. G., L. J. S. and H. L. under the supervision of M. J. W. A. G. L. carried out the molecular orbital calculations. The manuscript was written by M. U. and reviewed by M. J. W.

\section{Conflicts of interest}

There are no conflicts to declare.

\section{Acknowledgements}

We thank Newcastle University (SAgE doctoral training programme studentship award to MU), Cancer Research UK (C2115/A21421, Newcastle Drug Discovery Unit Programme Grant) and the Erasmus+ programme of the European Union (studentship award to LJS) for funding.

\section{Notes and references}

1 B. J. Drakulić, T. P. Stanojković, Ž. S. Žižak and M. M. Dabović, Eur. J. Med. Chem., 2011, 46, 3265-3273.

2 M. D. Vitorović-Todorović, A. Erić-Nikolić, B. Kolundžija, E. Hamel, S. Ristić, I. O. Juranić and B. J. Drakulić, Eur. J. Med. Chem., 2013, 62, 40-50.

3 A. Giordani, P. Pevarello, M. Cini, R. Bormetti, F. Greco, S. Toma, C. Speciale and M. Varasi, Bioorg. Med. Chem. Lett., 1998, 8, 2907-2912.

4 L. A. Arnold, A. Kosinski, E. Estébanez-Perpiñá and R. K. Guy, J. Med. Chem., 2007, 50, 5269-5280. 
5 M. Bianchi, A. Butti, Y. Christidis, J. Perronnet, F. Barzaghi, R. Cesana and A. Nencioni, Eur. J. Med. Chem., 1988, 23, 4552.

6 A. Dal Pozzo, M. Acquasaliente, G. Donzelli, P. De Maria and M. C. Nicoli, J. Med. Chem., 1987, 30, 1674-1677.

7 C. Xu, X. Bai, J. Xu, J. Ren, Y. Xing, Z. Li, J. Wang, J. Shi, L. Yu and Y. Wang, RSC Adv., 2017, 7, 4763-4775.

8 S. P. Annangudi, M. Sun and R. G. Salomon, Synlett, 2005, 2005, 1468-1470.

9 B. G. Sundar, T. Bailey, E. Bacon, L. Aimone, Z. Huang, J. Lyons, R. Raddatz and R. Hudkins, Bioorg. Med. Chem. Lett., 2011, 21, 5543-5546.

10 X. Li, N. Liu, H. Zhang, S. E. Knudson, H. J. Li, C. T. Lai, C. Simmerling, R. A. Slayden and P. J. Tonge, ACS Med. Chem. Lett., 2011, 2, 818-823.

11 J. Sun, L. Chen, C. Liu, Z. Wang, D. Zuo, J. Pan, H. Qi, K. Bao, Y. Wu and W. Zhang, Chem. Biol. Drug Des., 2015, 86, 15411547.

12 J. Ren, J. Xu, G. Zhang, C. Xu, L. L. Zhao, X. F. You, Y. Wang, Y. Lu, L. Yu and J. Wang, Bioorg. Med. Chem. Lett., 2019, 29, 539-543.

13 P. Jakubec, D. Berkeš, A. Kolarovič and F. Považanec, Synthesis, 2006, 4032-4040.

14 N. Chakor, S. Dallavalle, L. Musso and M. Moretti, Tetrahedron Lett., 2008, 49, 5056-5058.

15 W. L. Wan, J. B. Wu, F. Lei, X. L. Li, L. Hai and Y. Wu, Chin. Chem. Lett., 2012, 23, 1343-1346.

16 X. Wang, Y. Yan, B. Gong, X. Tang, Q. Li, Y. Meng, H. E. Xu and W. Yi, Bull. Korean Chem. Soc., 2013, 34, 3143-3146.

17 A. S. Amarasekara and U. Ha, Synth. Commun., 2018, 48, 2533-2538.

18 R. Scheffold and P. Dubs, Helv. Chim. Acta, 1967, 50, 798808.

19 R. Messer, A. Schmitz, L. Moesch and R. Häner, J. Org. Chem., 2004, 69, 8558-8560.

20 V. P. Ghidu, M. A. Ilies, T. Cullen, R. Pollet and M. AbouGharbia, Bioorg. Med. Chem. Lett., 2011, 21, 259-261.

21 A. Bolognese and G. Scherillo, J. Org. Chem., 1977, 42, 38673869.

22 K. Kaczanowska, H. Eickhoff, K. Albert, K. H. Wiesmüller and A. P. Schaffner, J. Heterocycl. Chem., 2011, 48, 792-798.

23 C. Desel, K. Werninghaus, M. Ritter, K. Jozefowski, J. Wenzel, N. Russkamp, U. Schleicher, D. Christensen, S. Wirtz, C. Kirschning, E. M. Agger, C. P. da Costa and R. Lang, PLoS One, 2013, 8, 1-9.
24 S. Zhao, J. B. Lin, Y. Y. Zhao, Y. M. Liang and P. F. Xu, Org. Lett., 2014, 16, 1802-1805.

25 M. A. I. Salem, M. I. Marzouk and A. M. El-Kazak, Molecules, 2016, 21, 249.

26 S. K. Wang, M. T. Chen, D. Y. Zhao, X. You and Q. L. Luo, Adv. Synth. Catal., 2016, 358, 4093-4099.

27 H. Chen, S. Zhao, S. Cheng, X. Dai, X. Xu, W. Yuan and X. Zhang, J. Heterocycl. Chem., 2019, 56, 1672-1683.

28 M. Lemurell, J. Ulander, H. Emtenäs, S. Winiwarter, J. Broddefalk, M. Swanson, M. A. Hayes, L. Prieto Garcia, A. Westin Eriksson, J. Meuller, J. Cassel, G. Saarinen, Z. Q. Yuan, C. Löfberg, S. Karlsson, M. Sundqvist and C. Whatling, J. Med. Chem., 2019, 62, 4325-4349.

29 M. Yamaguchi, N. Maruyama, T. Koga, T. Kuroki, K. Kamei, M. Hamana, M. Akima and N. Ohi, Chem. Pharm. Bull., 1994, 42, 1850-1853.

30 M. Krasavin, A. Shetnev, S. Baykov, S. Kalinin, A. Nocentini, V. Sharoyko, G. Poli, T. Tuccinardi, M. Korsakov, T. B. Tennikova and C. T. Supuran, Eur. J. Med. Chem., 2019, 168, 301-314.

31 J. Krall, C. H. Jensen, F. Bavo, C. B. Falk-Petersen, A. S. Haugaard, S. B. Vogensen, Y. Tian, M. NittegaardNielsen, S. B. Sigurdardóttir, J. Kehler, K. T. Kongstad, D. E. Gloriam, R. P. Clausen, K. Harpsøe, P. Wellendorph and B. Frølund, J. Med. Chem., 2017, 60, 9022-9039.

32 M. J. Frisch, G. W. Trucks, H. B. Schlegel, G. E. Scuseria, M. A. Robb, J. R. Cheeseman, G. Scalmani, V. Barone, G. A. Petersson, H. Nakatsuji, X. Li, M. Caricato, A. Marenich, J. Bloino, B. G. Janesko, R. Gomperts, B. Mennucci, H. P. Hratchian, J. V. Ortiz, A. F. Izmaylov, J. L. Sonnenberg, D. Williams-Young, F. Ding, F. Lipparini, F. Egidi, J. Goings, B. Peng, A. Petrone, T. Henderson, D. Ranasinghe, V. G. Zakrzewski, J. Gao, N. Rega, G. Zheng, W. Liang, M. Hada, M. Ehara, K. Toyota, R. Fukuda, J. Hasegawa, M. Ishida, T. Nakajima, Y. Honda, O. Kitao, H. Nakai, T. Vreven, K. Throssell, J. A. Montgomery, J. J. E. Peralta, F. Ogliaro, M. Bearpark, J. J. Heyd, E. Brothers, K. N. Kudin, V. N. Staroverov, T. Keith, R. Kobayashi, J. Normand, K. Raghavachari, A. Rendell, J. C. Burant, S. S. Iyengar, J. Tomasi, M. Cossi, J. M. Millam, M. Klene, C. Adamo, R. Cammi, J. W. Ochterski, R. L. Martin, K. Morokuma, O. Farkas, J. B. Foresman and D. J. Fox, Gaussian09, Revision D.02, Gaussian, Inc., Wallingford CT, 2016. 